\title{
固相烧结 $\mathrm{SiC}$ 陶瓷的非线性电学行为研究
}

\author{
陈 健, 殷 杰, 朱云洲, 杨勇, 陈忠明, 张景贤, 刘学建, 黄政仁 \\ (中国科学院 上海硅酸盐研究所, 结构陶瓷工程研究中心, 上海 200050)
}

摘 要: 固相烧结 $\mathrm{SiC}(\mathrm{SSiC})$ 陶瓷大多数用于结构陶瓷材料, 用于电子和电阻元器件的研究很少。实验以添加不同 $\mathrm{C}$ 含量的致密 $\mathrm{SSiC}$ 陶瓷材料为研究对象, 研究了添加不同 $\mathrm{C}$ 含量 $\mathrm{SSiC}$ 陶瓷的伏安特性、电阻率与电流密度的变化 关系及电阻率与温度的变化关系。研究结果表明: SSiC 陶瓷表现出明显的非线性电学特性, 其电阻率随着电流的增 大而降低; 对于添加 $3 \mathrm{wt} \% \mathrm{C}$ 含量的 $\mathrm{SSiC}$ 陶瓷，当电场强度超过 $15.8 \mathrm{~V} / \mathrm{mm}$ 时，晶界势垒被击穿; 对于添加 $6 \mathrm{wt} \% \mathrm{C}$ 含量的 SSiC 陶瓷，当电场强度超过 $70.7 \mathrm{~V} / \mathrm{mm}$ 时，晶界势垒被击穿，它们的电阻率将为晶粒所控制，电阻率较小; 同时在电场强度 $1 \mathrm{~V} / \mathrm{mm}$ 条件下, $\mathrm{SSiC}$ 陶瓷电阻率随着温度的升高而降低, 表现出很好热敏特性, 从常温的 $10^{6} \Omega^{\cdot} \mathrm{cm}$ 变化为 $400^{\circ} \mathrm{C}$ 的 $5 \Omega^{*} \mathrm{~cm}$ 左右。

关 键 词: $\mathrm{SiC}$; 固相烧结; 非线性电阻; 热敏电阻

中图分类号: TQ174 文献标识码: A

\section{Nonlinear Electrical Behavior of Solid State Sintered SiC Ceramics}

\author{
CHEN Jian, YIN Jie, ZHU Yun-Zhou, YANG Yong, CHEN Zhong-Ming, ZHANG Jing-Xian, \\ LIU Xue-Jian, HUANG Zheng-Ren
}

(Structural Ceramics Engineering Research Center, Shanghai Institute of Ceramics, Chinese Academy of Sciences, Shanghai 200050, China)

\begin{abstract}
The solid state sintered $\mathrm{SiC}(\mathrm{SSiC})$ ceramics are mainly used for structural materials, but they are rarely used for electronic devices and resistance components. In this work, the electrical properties of the SSiC ceramics with different carbon additives were investigated, including $V-I$ characteristic, the relationship between current and resistance, as well as the relationship between temperature and resistance. These results show that the SSiC ceramics present obvious nonlinear electrical behavior and their resistance decreases with the current increase. When the electrical field intensity is over $15.8 \mathrm{~V} / \mathrm{mm}$ for the $\mathrm{SSiC}$ ceramics with $3 \mathrm{wt} \%$ carbon additives, as well as $70.7 \mathrm{~V} / \mathrm{mm}$ for the $\mathrm{SSiC}$ ceramics with $6 \mathrm{wt} \%$ carbon additives, the boundary barrier is breakdown and the resistance becomes very low, due to the resistance being only controlled by the grain size. The resistance of the SSiC ceramics decreases with the temperature increase at the electrical field intensity of $1 \mathrm{~V} / \mathrm{mm}$, indicating thermo-sensitive characteristic of SSiC ceramics of which the resistance is changed from $10^{6} \Omega \cdot \mathrm{cm}$ at $25^{\circ} \mathrm{C}$ to $5 \Omega \cdot \mathrm{cm}$ at $400^{\circ} \mathrm{C}$.
\end{abstract}

Key words: $\mathrm{SiC}$; solid state sintered; nonlinear resistance; thermistor

在工业应用中，越来越多的传感器和致动器部 署在温度较高的环境中, 标准的半导体和元件所能
承受的温度最高约为 $125^{\circ} \mathrm{C}$, 因此业界迫切需要新 的元件具有更高的封装密度，并可在高达 $300^{\circ} \mathrm{C}$

收稿日期: 2016-03-23; 收到修改稿日期：2016-05-19

基金项目: 国家自然科学基金(51302288) National Natural Science Foundation of China (51302288)

作者简介：陈 健(1981-), 男, 副研究员. E-mail: nannancj@mail.sic.ac.cn

通讯作者: 黄政仁, 研究员. E-mail: zhrhuang@mail.sic.ac.cn 
$\left(572^{\circ} \mathrm{F}\right)$ 的温度下可靠地工作。固相烧结碳化硅 (Solid state sintered $\mathrm{SiC}-\mathrm{SSiC}$ )陶瓷的烧结助剂含量 较低, 具有高温稳定性, 耐强酸强碱环境等特性, 常应用于机械工业、汽车和化工领域中的轴承、活 塞及密封环等部件 ${ }^{[1-5]}$, 很少应用于电子元器件行 业，而 $\mathrm{SiC}$ 材料以其材料本身所具备的优势而被业 界称为 “极端电子学器件” 的基础材料 ${ }^{[6]}$, 它具有半 导体特性, 临界击穿电场和热导率高, 介电常数低, 是制造高温、高辐射条件下工作的高频大功率器件 和高功率密度、高集成度器件的优良材料 ${ }^{[7-12]}$ 。 $\mathrm{SiC}$ 材料具有非线性电阻特性, 主要包括热敏特性和压 敏特性。目前实用化的 $\mathrm{SiC}$ 电子器件主要有 $\mathrm{SiC}$ 半 导体材料和热敏电阻 ${ }^{[13-16]}$, 其中半导体材料主要通 过射频溅射方法获得的薄膜型和升华法制备的单晶 型材料, 这些电子器件的制作成本非常高而且也无 法通过设计获得不同形状和尺寸较大的材料。文献 [16]报道的热敏电阻主要是简单的重结晶 $\mathrm{SiC}$, 没 有添加任何烧结助剂, 材料无法高温致密化, 耐腐 蚀性能和力学性能相对较差。而通过粉体烧结致密 化方法获得的 SSiC 陶瓷材料, 其制作方法简单, 形状和尺寸可以根据需要进行设计, 制作成本较低, 可以规模化生产等特点。目前对于 $\mathrm{SiC}$ 非线性电阻 陶瓷的研究很少, 而且研究得不够深入, 文献 [17-20]通过在 $\mathrm{SiC}$ 粉体中掺杂第二相的方法获得高 电阻的 $\mathrm{SiC}$ 复相陶瓷, 但是陶瓷性能是在低电场强 度下测试的, 没有继续研究其伏安特性和耐电场强 度特性, 而这些研究将为获得高电阻晶界相提供研 究基础 ${ }^{[21]}$ 。结合 SSiC 陶瓷材料优良的物理化学性 能, 本工作通过深入研究其电学性能, 包括伏安特 性和电阻率随温度的变化关系等, 从而对 $\mathrm{SSiC}$ 的 电学性能有深入的了解, 为拓展 $\mathrm{SSiC}$ 陶瓷的应用 领域提供理论支持和奠定基础。

\section{1 实验方法}

实验原料选用平均粒径为 $0.5 \mu \mathrm{m}$ 的 $\mathrm{SiC}$ 粉体 (FCP-1, Norton Co, Lillesand, Norway), 碳化喼 $\left(\mathrm{B}_{4} \mathrm{C}\right)$ 和纳米碳黑作为烧结助剂, 酚醛树脂作为成 型粘结剂。将上述原料 $\mathrm{SiC}$ 粉体、 $\mathrm{B}_{4} \mathrm{C}-0.5 \mathrm{wt} \%$ (占 粉体总量)、与纳米碳黑按照比例加入到无水乙醇 中, 控制酚醛树脂裂解碳为粉体质量的 $3 \mathrm{wt} \%$, 纳 米碳黑添加量分别为粉体质量的 $0 、 1 \mathrm{wt} \% 、 2 \mathrm{wt} \%$ 、 $3 \mathrm{wt} \%$, 两者碳添加总量分别为粉体总质量的 $3 \mathrm{wt} \% 、 4 \mathrm{wt} \% 、 5 \mathrm{wt} \% 、 6 \mathrm{wt} \%$ 。原料球磨混合后干 燥, 过篎后经 $40 \mathrm{MPa}$ 干压、200 MPa 等静压成型,
成型样品经 $1000^{\circ} \mathrm{C}$ 脱粘后, 再在高温烧结炉中经 $2200^{\circ} \mathrm{C}$ 保温 $1 \mathrm{~h}$ 烧结。

采用阿基米德法测定烧结后 $\mathrm{SSiC}$ 陶瓷样品的 密度。采用三点弯曲法在 Instron 5566 材料试验机 测试 $\mathrm{SSiC}$ 陶瓷样品的力学性能, 样品尺寸为 $3 \mathrm{~mm} \times 4 \mathrm{~mm} \times 36 \mathrm{~mm}$, 加载速率为 $0.5 \mathrm{~mm} / \mathrm{min}$, 性 能数据为 5 根试样数据的平均值。采用 Olympus 光 学显微镜和扫描电镜 (Scanning electron microscopy-SEM) 观察 SSiC 陶瓷微观结构, 观察前将样品 抛光并经熔融 $\mathrm{NaOH}$ 腐蚀。测定 $\mathrm{SSiC}$ 陶瓷电学性 能之前, 对其表面进行高温银浆电极处理。采用 Agilent4294A 精密阻抗分析仪获得 Nyquist 图来分 析 SSiC 陶瓷的等效电路图, 采用 Keithley2450 多通 道测试系统测试 SSiC 陶瓷伏安特性曲线, 通过将 马弗炉升温 SSiC 陶瓷材料, 并采用 Keithley2450 多 通道测试系统测定 $\mathrm{SSiC}$ 陶瓷电阻率与温度的变化 关系。

\section{2 结果与讨论}

\section{1 不同 C 含量 SSiC 陶瓷力学性能与微观结构}

添加不同 $\mathrm{C}$ 含量的 $\mathrm{SSiC}$ 陶瓷样品经 $2200^{\circ} \mathrm{C}$ 的 高温烧结, 获得的样品烧结都比较致密, 力学性能 良好。随着添加 $\mathrm{C}$ 含量的增多, 其抗弯强度增加(如 表 1 所示), 主要由于 $\mathrm{C}$ 抑制了晶粒的长大, 随着 $\mathrm{C}$ 含量的增加, 晶粒减小, 如图 1 所示。 $\mathrm{C}$ 作为 $\mathrm{SSiC}$ 陶瓷中常用的烧结助剂, 添加的 $\mathrm{C}$ 含量在 $3 \mathrm{wt} \%$ $6 \mathrm{wt} \%$ 变化时, 可以获得高致密度和优良力学性能 的 $\mathrm{SSiC}$ 陶瓷材料, 同时烧结后 $\mathrm{C}$ 以第二相形式分布 于 $\mathrm{SSiC}$ 陶瓷中，没有形成联通的相，如图 2 所示。

\section{2 不同 C 含量 SSiC 陶瓷的等效电路图和电} 阻率变化规律

通过交流阻抗法对不同 $\mathrm{C}$ 含量的 $\mathrm{SSiC}$ 陶瓷进 行测试, 测试频率范围为 $40 \mathrm{~Hz} 110 \mathrm{MHz}$, 电场强 度为 $0.1 \mathrm{~V} / \mathrm{mm}$, 结果如图 3 所示。其测试结果符合 以下公式 ${ }^{[22]}$ :

表 1 不同 C 含量 SSiC 陶瓷室温下的性能 Table 1 Properties of SiC with different $C$ contents at room temperature

\begin{tabular}{cccc}
\hline C additives & $\begin{array}{c}\text { Density/ } \\
\left(\mathrm{g} \cdot \mathrm{cm}^{-3}\right)\end{array}$ & $\begin{array}{c}\text { Relative } \\
\text { density/\% }\end{array}$ & $\begin{array}{c}\text { Flexural } \\
\text { strength/MPa }\end{array}$ \\
\hline $3 \mathrm{wt} \%$ & $3.08 \pm 0.03$ & $97 \%$ & $303.7 \pm 17.3$ \\
$4 \mathrm{wt} \%$ & $3.09 \pm 0.02$ & $98 \%$ & $378.3 \pm 10.1$ \\
$5 \mathrm{wt} \%$ & $3.09 \pm 0.01$ & $98 \%$ & $405.4 \pm 19.2$ \\
$6 \mathrm{wt} \%$ & $3.11 \pm 0.01$ & $99 \%$ & $486.2 \pm 20.4$ \\
\hline
\end{tabular}



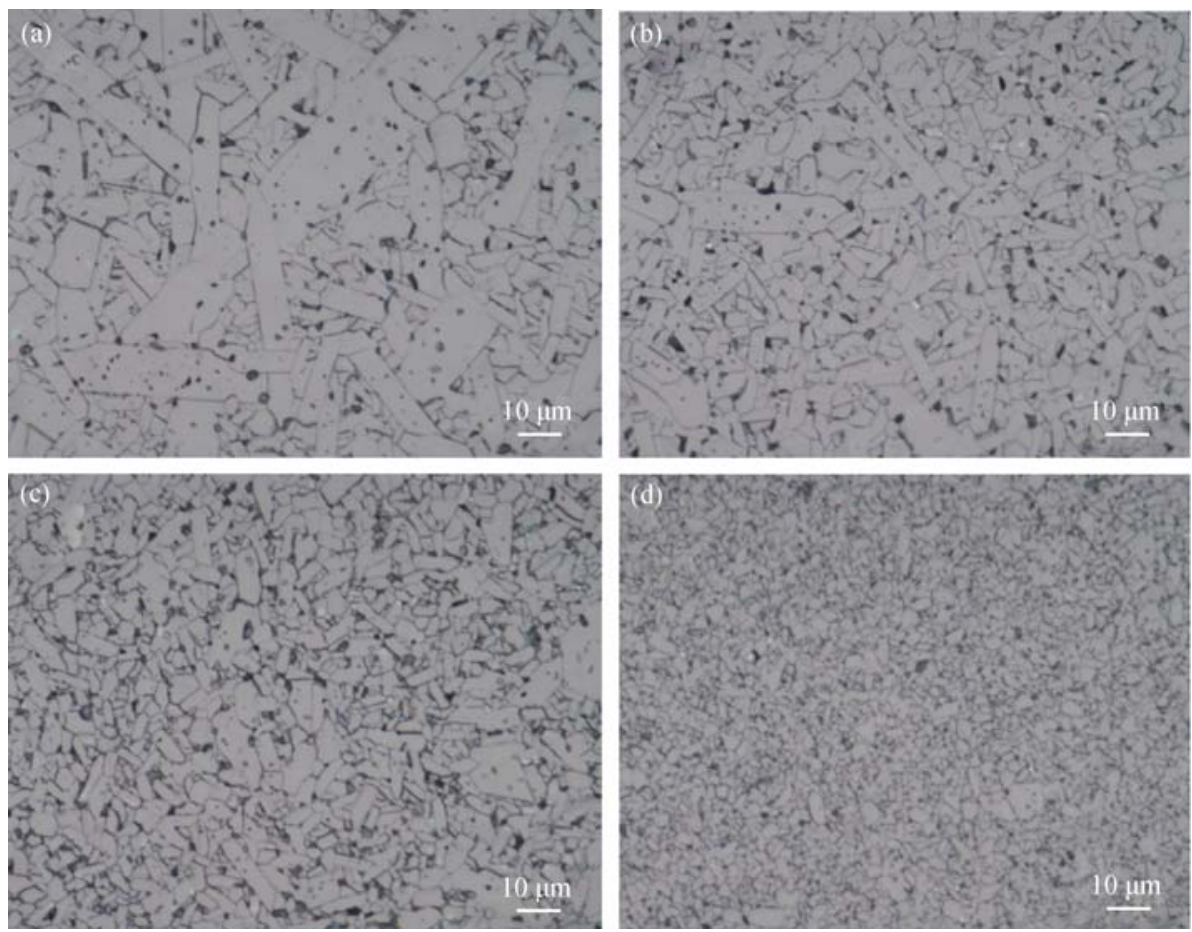

图 1 不同 $\mathrm{C}$ 含量 $\mathrm{SSiC}$ 陶瓷的微观结构

Fig. 1 Microstructure of SSiC with different $\mathrm{C}$ contents (a) $3 \mathrm{wt} \%$; (b) $4 \mathrm{wt} \%$; (c) $5 \mathrm{wt} \%$; (d) $6 \mathrm{wt} \%$
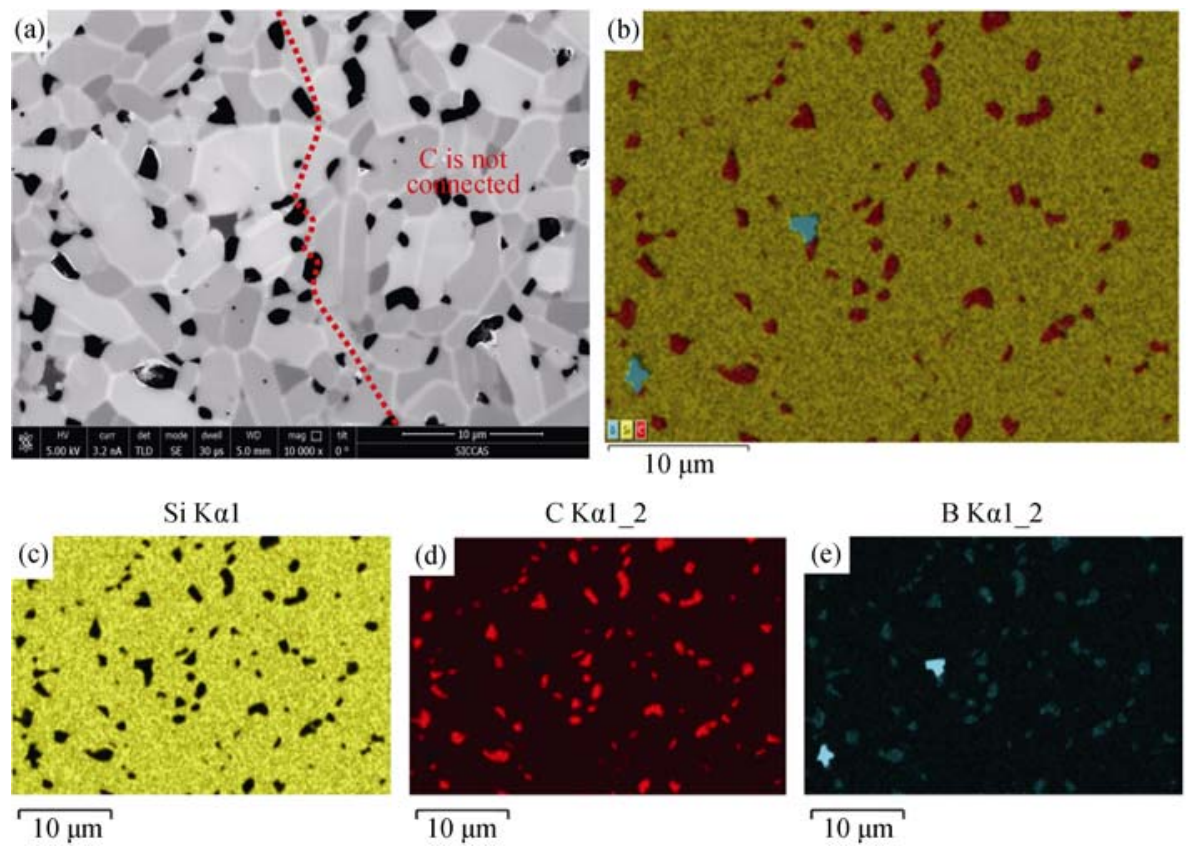

图 $26 \mathrm{wt} \% \mathrm{C}-\mathrm{SSiC}$ 陶瓷 SEM 照片(a)和元素扫描图(b-Si/C/B, c-Si, d-C, e-B)

Fig. 2 SEM images (a) and element distribution (b-Si/C/B, c-Si, d-C, e-B) of $6 \mathrm{wt} \% \mathrm{C}-\mathrm{SSiC}$ ceramics

$$
\begin{gathered}
\left(\mathrm{Z}^{\prime}-R_{\mathrm{G}}-\frac{R_{\mathrm{B}}}{2}\right)^{2}+Z^{\prime \prime 2}=\left(\frac{R_{\mathrm{B}}}{2}\right)^{2} \\
\mathrm{Z}^{\prime}=R_{\mathrm{G}}+\frac{R_{\mathrm{B}}}{1+R_{\mathrm{B}}^{2} \omega^{2} C^{2}} \\
\mathrm{Z}^{\prime \prime}=-\frac{\omega R_{\mathrm{B}}^{2} C}{1+R_{\mathrm{B}}^{2} \omega^{2} C^{2}}
\end{gathered}
$$

$\mathrm{Z}^{\prime}$ 为交流阻抗实部, $\mathrm{Z}^{\prime \prime}$ 为交流阻抗虚部, $R_{\mathrm{G}}$ 为晶粒 电阻率, $R_{\mathrm{B}}$ 为晶界电阻率, $C$ 为电容, $\omega$ 为电流频率。 根据其等效电路图, $\mathrm{SSiC}$ 陶瓷阻抗主要由晶粒电阻 率和晶界电阻率，以及与晶界电阻率并联的晶界电 容所组成。当频率趋于 0 时，陶瓷的阻抗为晶粒电 阻率与晶界电阻率之和; 当频率趋于无穷大 

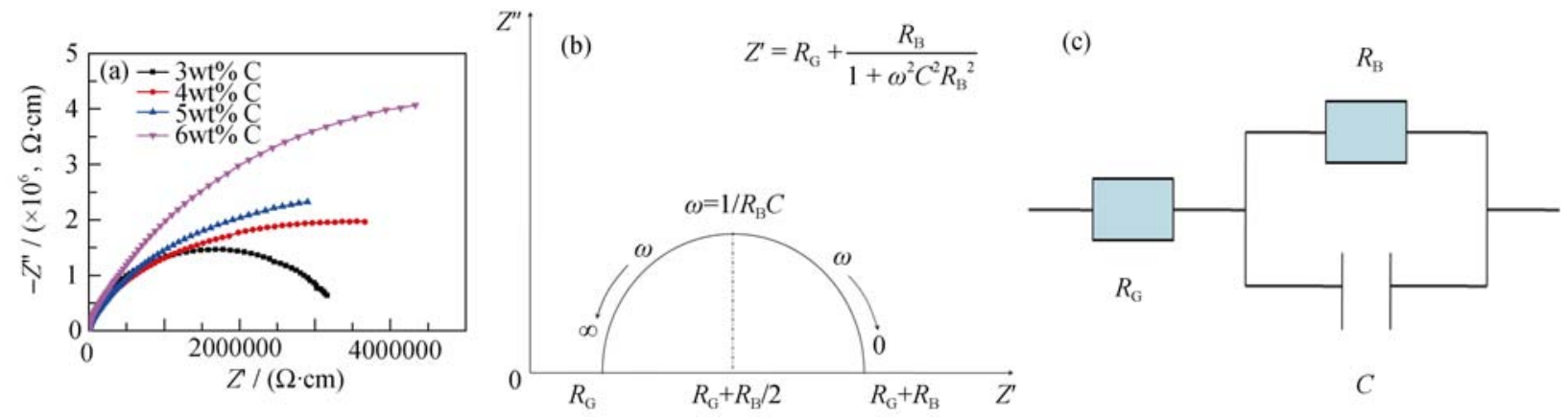

图 3 不同含量 $\mathrm{C}$ 的 $\mathrm{SSiC}$ 陶瓷的 Nyquist 图谱(a)和理想的 Nyquist 图谱(b)及其等效电路图(c)

Fig. 3 Nyquist plots of SSiC ceramics with different C contents (a), theory Nyquist plots (b) and equivalent circuits (c).

时, 陶瓷的阻抗仅仅由晶粒电阻率决定, 即 $\omega \rightarrow \infty$, $\mathrm{Z}^{\prime}=R_{\mathrm{G}}$ 。并由此可以推测 $\mathrm{SSiC}$ 陶瓷的晶粒电阻率 大小如表 2 所示。

由表 2 可以看出晶粒电阻率较小, 随着 $\mathrm{C}$ 含量 增加, 晶粒电阻率变化不大, 主要是由于 $\mathrm{B}_{4} \mathrm{C}$ 固溶 于 $\mathrm{SiC}$ 中, B 元素掺杂取代 $\mathrm{Si}$ 的位置, 形成空位, $\mathrm{SiC}$ 晶粒变成 $\mathrm{P}$ 型半导体。晶界电阻率很大, 是由 于晶界电阻率主要由肖特基势垒 $\Phi_{\mathrm{B}}$ 所决定, 如公 式(4)所示:

$$
R_{\mathrm{B}}=R_{0} \exp \left(\frac{\mathrm{e} \Phi_{\mathrm{B}}}{\mathrm{k} T}\right)
$$

其中 $R_{0}$ 为肖特基势垒 $\Phi_{\mathrm{B}}$ 为零时材料的电阻率。

当 $\omega \rightarrow 0, \mathrm{Z}^{\prime}=R_{\mathrm{B}}+R_{\mathrm{G}}$, 由图 3 可以看出: 随着 $\mathrm{C}$ 含量的增加, $\mathrm{SSiC}$ 陶瓷的电阻率增大, 主要是由于 随着 $\mathrm{C}$ 含量的增多, 晶粒减小, 晶界数量增多, 肖 特基势垒 $\Phi_{\mathrm{B}}$ 增大。由于 $R_{\mathrm{B}}>>R_{\mathrm{G}}$, 不同 $\mathrm{C}$ 含量 $\mathrm{SSiC}$ 陶瓷的电阻率 $R=R_{\mathrm{B}}+R_{\mathrm{G}}$ 主要取决于晶界电阻率 $\mathrm{R}_{\mathrm{B}}$, 而且与 $\mathrm{C}$ 含量增加无关, 因为第二相 $\mathrm{C}$ 并没有形成 联通相(如图 2), 因此电阻率 $R(\omega=0)$ ) 随着 $\mathrm{C}$ 含量增 加反而增大:

$$
R_{(6 \mathrm{wt} \% \mathrm{C})}>R_{(5 \mathrm{wt} \% \mathrm{C})}>R_{(4 \mathrm{w} \% \mathrm{C})}>R_{(3 \mathrm{w} w \% \mathrm{C})}
$$

\subsection{SSiC 陶瓷的伏安特性和非线性系数}

通过 Keithley2450 系统测试不同 C 含量下 $\mathrm{SiC}$ 陶瓷的伏安特性曲线, 如图 4 所示。从图 4 可以看 出, 表现明显的非线性特性, 随着电场强度 $\mathrm{U}$ 的增 加, 电流密度 $I$ 非线性增加。对于添加 $3 \mathrm{wt} \% \mathrm{C}$ 含量 $\mathrm{SSiC}$ 陶瓷, 陶瓷的电阻率随着电流密度的增加而减 小, 当电场强度超过 $15.8 \mathrm{~V} / \mathrm{mm}$ 时, 继续增大电流

表 2 不同 C 含量 SSiC 陶瓷晶粒电阻率

Table 2 Grain resistance of SSiC ceramics with different carbon contents

\begin{tabular}{ccccc}
\hline $\mathrm{C}$ & $3 \mathrm{wt} \%$ & $4 \mathrm{wt} \%$ & $5 \mathrm{wt} \%$ & $6 \mathrm{wt} \%$ \\
\hline$R_{\mathrm{G}} /(\Omega \cdot \mathrm{cm})$ & 150 & 148 & 146 & 48 \\
\hline
\end{tabular}

密度，场强降低，电阻率降低; 对于添加 $6 \mathrm{wt} \% \mathrm{C}$ 含 量 $\mathrm{SSiC}$, 陶瓷的电阻率随着电流密度的增加而减小, 当电场强度达到 $70.7 \mathrm{~V} / \mathrm{mm}$ 时, 继续增大电流密度, 电场强度会降低, 电阻率同样继续降低。由等效电 路图可以看出, 在电场强度超过一定值时, 晶界势 垒被击穿, 此时电阻率主要由晶粒电阻率所决定, 而晶粒电阻率非常小, 如表 2 所示。根据公式(5)H 为位形系数, 与非线性电阻的体积形状、电阻片的 串、并联组合以及材质有关):

$$
I=H U^{\alpha}
$$

不同 $\mathrm{C}$ 含量 $\mathrm{SiC}$ 陶瓷的非线性系数 $\alpha$ 为:

$$
\alpha=\frac{\mathrm{d} \lg I}{\mathrm{~d} \lg U}
$$

由表 3 可以看出, 随着 $\mathrm{C}$ 含量的增加, $\mathrm{SSiC}$ 陶 瓷非线性系数减小, 而压敏电压 $\left(1 \mathrm{~mA} / \mathrm{cm}^{2}\right)$ 在升高, 主要是由于 $\mathrm{C}$ 含量的增加, 晶粒减小, 晶界数量增 多, 肖特基势垒高度在增加, 相应晶界电阻率增大, 对于 $\mathrm{SSiC}$ 而言晶界干净, 晶界宽度基本没有变化, 因此非线性系数在减小, 压敏电压在增大。

\section{$2.4 \mathrm{SSiC}$ 陶瓷的热敏特性}

在 $1 \mathrm{~V} / \mathrm{mm}$ 的电场强度下测定 $\mathrm{SSiC}$ 陶瓷电阻率 随温度的变化关系, 由图 5 可以看出 $\mathrm{SSiC}$ 陶瓷电阻 率随着温度升高而降低, 表现为很好热敏特性, 从 常温的 $10^{6} \Omega \cdot \mathrm{cm}$ 变化为 $400^{\circ} \mathrm{C}$ 的 $5 \Omega \cdot \mathrm{cm}$ 左右。通过 对图 5 曲线分析, 在 $220^{\circ} \mathrm{C}$ 以下的低温温度段有两 个温度阶段电阻率的对数与温度的倒数表现为线性

表 3 不同 C 含量 SSiC 陶瓷非线性系数和压敏电压

Table 3 Non-linear coefficient and varistor voltage of SSiC ceramics

\begin{tabular}{ccccc}
\hline $\mathrm{C}$ & $3 \mathrm{wt} \%$ & $4 \mathrm{wt} \%$ & $5 \mathrm{wt} \%$ & $6 \mathrm{wt} \%$ \\
\hline$\alpha$ & 1.80 & 1.60 & 1.44 & 1.16 \\
$U_{1 \mathrm{~mA}} /\left(\mathrm{V} \cdot \mathrm{mm}^{-1}\right)$ & 2.2 & 3.7 & 4.2 & 10.6 \\
\hline
\end{tabular}



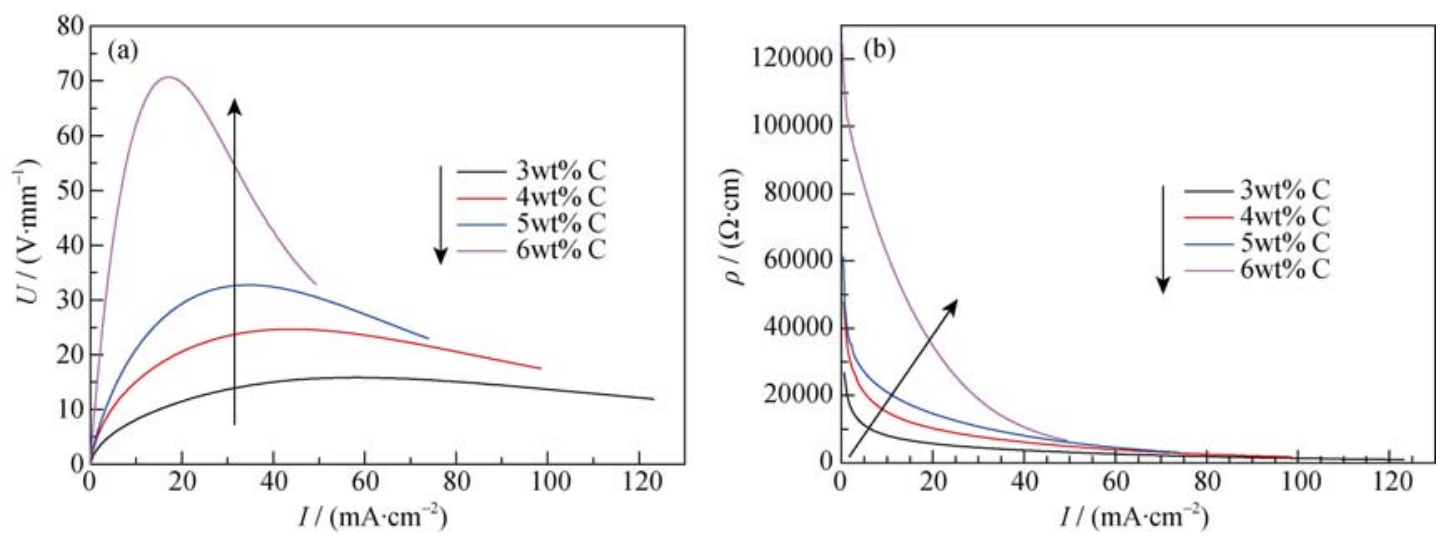

图 4 不同 $\mathrm{C}$ 含量 $\mathrm{SSiC}$ 陶瓷伏安特性曲线(a)及电阻率随电流密度变化曲线(b)

Fig. $4 V$-I curves of SSiC ceramics with different $\mathrm{C}$ contents (a) and relationship between current density and resistance (b)
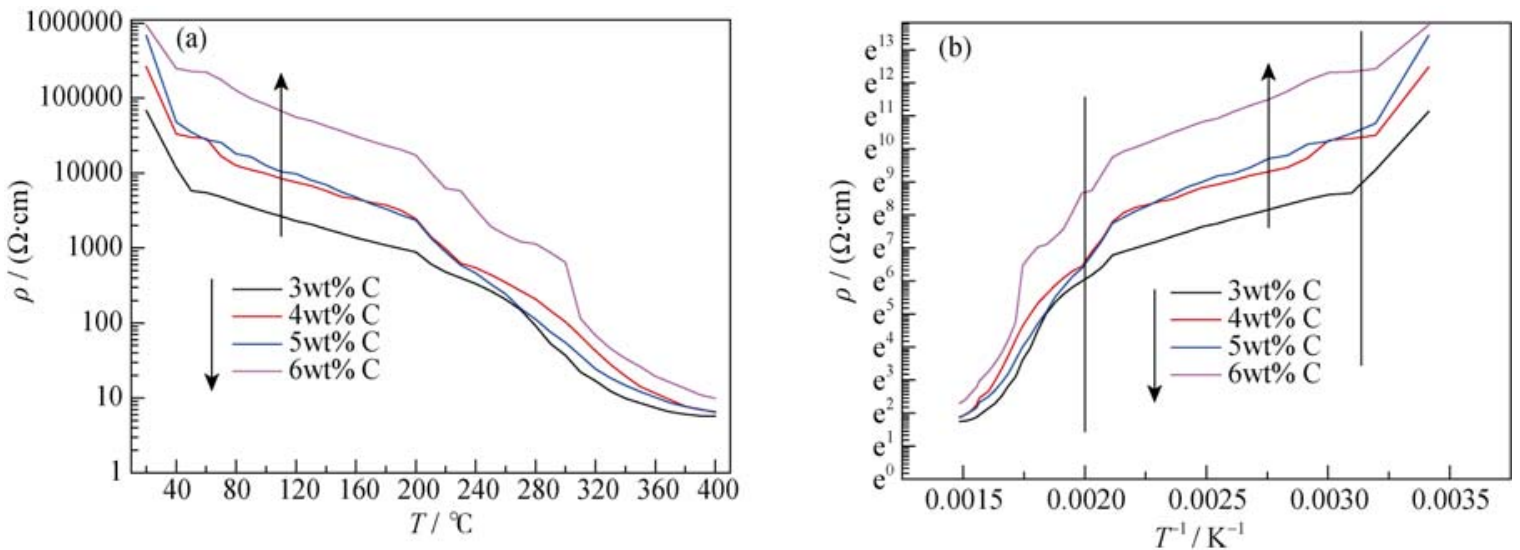

图 5 不同 $\mathrm{C}$ 含量 $\mathrm{SSiC}$ 陶瓷的热敏特性( $\mathrm{a}, \mathrm{b}$-取对数)

Fig. 5 Thermosensitivity of SSiC ceramics with different $\mathrm{C}$ contents (a, b- logarithmic)

关系, 即材料电阻率的对数与温度的倒数符合 Arrhenius 公式:

$$
\begin{gathered}
\ln R_{\mathrm{B}}=\ln R_{0}+\frac{\mathrm{e} \Phi_{\mathrm{B}}}{\mathrm{k}}\left(\frac{1}{T}\right) \\
\ln R_{\mathrm{B}}=\ln R_{0}+\beta\left(\frac{1}{T}\right)
\end{gathered}
$$

( $\beta$ 为材料的敏感指数)

而在大于 $220^{\circ} \mathrm{C}$ 的高温阶段, 材料电阻率的对 数与温度的倒数不符合 Arrhenius 公式, 因为在高温 阶段, 材料晶界被击穿, 此时陶瓷材料的电阻率主 要由晶粒控制, 因此不能用 Arrhenius 公式来解释, 这也证明了之前的分析。

\section{3 结论}

1) SSiC 陶瓷表现出明显的非线性电学行为, 通 过控制电压和温度可以调控 $\mathrm{SSiC}$ 陶瓷的电阻率;

2) 随着添加 C 含量的增加, SSiC 陶瓷电阻率增 加, $\mathrm{SSiC}$ 陶瓷中第二相 $\mathrm{C}$ 没有形成联通的相, 因此
$\mathrm{SSiC}$ 陶瓷电阻率与第二相 $\mathrm{C}$ 的存在没有直接关系, 但是由于随着添加 $\mathrm{C}$ 含量的增加, 晶粒减小, 晶界增 多, 晶界势垒增大, 导致陶瓷电阻率增加;

3) $\mathrm{SSiC}$ 陶瓷随着添加 $\mathrm{C}$ 含量的增加, 压敏电压 增加, 非线性系数减小, 压敏特性减小;

4) $\mathrm{SSiC}$ 陶瓷电阻率随着电流密度的增加而降 低, 对于添加 $3 \mathrm{wt} \% \mathrm{C}-\mathrm{SSiC}$ 陶瓷, 当电场强度超过 $15.8 \mathrm{~V} / \mathrm{mm}$ 时, $\mathrm{SSiC}$ 陶瓷晶界势垒被击穿; 对于添 加 $6 \mathrm{wt} \% \mathrm{C}-\mathrm{SSiC}$ 陶瓷, 当电场强度超过 $70.7 \mathrm{~V} / \mathrm{mm}$ 时, $\mathrm{SSiC}$ 陶瓷晶界势垒被击穿, 它们的电阻率将为 晶粒所控制，电阻率较低;

5) 在电场强度 $1 \mathrm{~V} / \mathrm{mm}$ 条件下, $\mathrm{SSiC}$ 陶瓷电 阻率随着温度的升高而降低，表现为很好的热敏 特性, 从常温的 $10^{6} \Omega \cdot \mathrm{cm}$ 变化为 $400^{\circ} \mathrm{C}$ 的 $5 \Omega \cdot \mathrm{cm}$ 左右;

总体而言, $\mathrm{SSiC}$ 陶瓷具有非线性电学特性, 可 以作为低压压敏陶瓷和热敏陶瓷使用，但是非线性 系数偏小, 还有很大的提高空间, 有待进一步深入 的研究。 


\section{参考文献:}

[1] CHEN J, HUANG Z R, CHEN Z M, et al. The effect of carbon on surface quality of solid state sintered silicon carbide as optical materials. Mater. Charact., 2014, 89: 7-12.

[2] GAO J Q, CHEN J, LIU G L, et al. Improvement on surface quality of solid sintering SiC ceramics. Int. J. Appl. Ceram. Technol., 2012, 9(4): 847-852.

[3] GAO J Q, CHEN J, LIU G L, et al. Role of microstructure on surface and subsurface damage of sintered silicon carbide during grinding and polishing. Wear, 2010, 270(1/2): 88-94.

[4] 江东亮, 李龙土, 欧阳世翕等主编. 中国材料工程大典(第 8 卷)(无机非金属材料). 北京: 化学工业出版社, 2006 .

[5] LI Y S, YIN J, WU H B, et al. High thermal conductivity in pressureless densified $\mathrm{SiC}$ ceramics with ultra-low contents of additives derived from novel boron-carbon sources. J. Eur. Ceram. Soc., 2014, 34(10): 2591-2595.

[6] ZHANG Y M, TANG X Y, SONG Q W. Status of SiC power semiconductor devices. Advanced Materials Industry, 2015, 10: 26-31.

[7] QIAN Z M, ZHANG J M, SHENG K. Status and development of power semiconductor devices and its applications. Chin. Soc. for Elec. Eng., 2014, 34(29): 5149-5161.

[8] WANG X M. Researches and applications of wide band gap SiC power devices in electric vehicles. Chin. Soc. for Elec. Eng., 2014, 34(3): 371-378.

[9] SHENG K, GUO Q, ZHANG J M et al. Development and prospect of $\mathrm{SiC}$ power devices in power grid. Chin. Soc. for Elec. Eng., 2012, 32(30): 1-7.

[10] ZHAO M, XU H Y, YANG Q, et al. Research of packaging technology on $\mathrm{SiC}$ power devices under high temperature and high voltage. Smart Grid, 2014, 4: 252-258.

[11] SON N T,CARLSSON P,GALLSTROM A, et al. Deep levels responsible for semi-insulating behavior in vanadium-doped $4 \mathrm{H}-\mathrm{SiC}$ substrates. Mater. Sci. Forum., 2009, 600-603(1): 401-404.
[12] SON N T, CARLSSON P, GALLSTROM A, et al. Prominent defects in semi-insulating SiC substrates. Physica B: Condensed Matter, 2007, 401-402(1): 67-72.

[13] TODOROVA Z, DISHOVSKY N, DIMITROV R, et al. Natural rubber filled $\mathrm{SiC}$ and $\mathrm{B}_{4} \mathrm{C}$ ceramic composites as a new NTC thermistors and piezoresistive sensor materials. Polym. Compos., 2008, 1(29): 109-118.

[14] GUO L, NING S F, YU K K, et al. Study progress of silicon carbide nonlinear property. Insulating Materials, 2005, 3: 60-64.

[15] ROBERTSO J. The Application of METROSIL ${ }^{\circledR S i C}$ nonlinear Resistance in De-excitation System. Proceedings of the 2nd International conference on Hydraulic Power Generation Technology, 2005.

[16] QIU S C, HUANG H R, XU Y Z, et al. The conduction mechanism of $\mathrm{SiC}$ ceramic thermistors. J. Huangzhong. Univ. of Sci. \& Tech., 1992, 20(6): 103-108.

[17] LI Y S, YIN J, WU H B, et al. Enhanced electrical resistivity in SiC-BN composites with highly-active BN nanoparticles synthesized via chemical route. J. Eur. Ceram. Soc., 2015, 35(5): 1647-1652.

[18] LI Y S, YIN J, WU H B, et al. Microstructure, Thermal conductivity, and electrical properties of in situ pressureless densified SiC-BN Composites. J. Am. Ceram. Soc., 2015, 98(3): 879-887.

[19] LIANG H Q, YAO X M, HAO D, et al. High electrical resistivity of spark plasma sintered $\mathrm{SiC}$ ceramics with $\mathrm{Al}_{2} \mathrm{O}_{3}$ and $\mathrm{Er}_{2} \mathrm{O}_{3}$ as sintering additives. J. Eur. Ceram. Soc., 2015, 35: 399-403.

[20] LI Y S, WU H B, YIN J, et al. High electrical resistivity of pressureless sintered in situ SiC-BN composites. Scr. Mater, 2013, 69(10): 740-743.

[21] BUENO P R, LEITE E R,OLIVEIRA M M, et al. Role of oxygen at the grain boundary of metal oxide varistors: a potential barrier formation mechanism. Appl. Phys. Lett., 2001,79: 48-50.

[22] CONWAY B E, BOCKRIS J O M, WHITE R E, et al. Electrochemistry Impedance Spectroscopy and Its Application: Modern Aspects of Electrochemistry. New York: Kluwer Academic Publishers, 1999, 32: 143-348. 